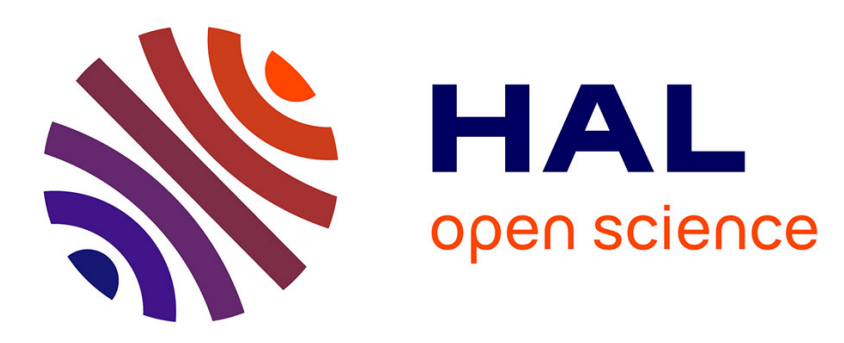

\title{
Coherent Scattering of Near-Resonant Light by a Dense Microscopic Cold Atomic Cloud
}

\author{
Stephan Jennewein, Mondher Besbes, N J Schilder, S. d. D Jenkins, \\ Christophe Sauvan, J. Ruostekoski, J.-J. Greffet, Yvan R. P. Sortais, Antoine
} Browaeys

\section{To cite this version:}

Stephan Jennewein, Mondher Besbes, N J Schilder, S.d. D Jenkins, Christophe Sauvan, et al.. Coherent Scattering of Near-Resonant Light by a Dense Microscopic Cold Atomic Cloud. Physical Review Letters, 2016, 116 (23), pp.233601. 10.1103/PhysRevLett.116.233601 . hal-01643262

\section{HAL Id: hal-01643262 https://hal.science/hal-01643262}

Submitted on 21 Nov 2017

HAL is a multi-disciplinary open access archive for the deposit and dissemination of scientific research documents, whether they are published or not. The documents may come from teaching and research institutions in France or abroad, or from public or private research centers.
L'archive ouverte pluridisciplinaire HAL, est destinée au dépôt et à la diffusion de documents scientifiques de niveau recherche, publiés ou non, émanant des établissements d'enseignement et de recherche français ou étrangers, des laboratoires publics ou privés. 


\title{
Coherent Scattering of Near-Resonant Light by a Dense Microscopic Cold Atomic Cloud
}

\author{
S. Jennewein, ${ }^{1}$ M. Besbes, ${ }^{1}$ N. J. Schilder, ${ }^{1}$ S. D. Jenkins, ${ }^{2}$ C. Sauvan, ${ }^{1}$ J. Ruostekoski, ${ }^{2}$ J.-J. Greffet, ${ }^{1}$ \\ Y. R. P. Sortais, ${ }^{1}$ and A. Browaeys ${ }^{1, *}$ \\ ${ }^{1}$ Laboratoire Charles Fabry, Institut d'Optique Graduate School, CNRS, Université Paris-Saclay, \\ 91127 Palaiseau cedex, France \\ ${ }^{2}$ Mathematical Sciences, University of Southampton, Southampton SO17 1BJ, United Kingdom
}

(Received 27 October 2015; published 8 June 2016)

\begin{abstract}
We measure the coherent scattering of light by a cloud of laser-cooled atoms with a size comparable to the wavelength of light. By interfering a laser beam tuned near an atomic resonance with the field scattered by the atoms, we observe a resonance with a redshift, a broadening, and a saturation of the extinction for increasing atom numbers. We attribute these features to enhanced light-induced dipole-dipole interactions in a cold, dense atomic ensemble that result in a failure of standard predictions such as the "cooperative Lamb shift". The description of the atomic cloud by a mean-field model based on the Lorentz-Lorenz formula that ignores scattering events where light is scattered recurrently by the same atom and by a microscopic discrete dipole model that incorporates these effects lead to progressively closer agreement with the observations, despite remaining differences.
\end{abstract}

DOI: 10.1103/PhysRevLett.116.233601

The understanding of light propagation in dense media relies traditionally on a continuous description of the sample characterized by macroscopic quantities such as susceptibility or refractive index [1,2]. Their derivation from a microscopic theory is in general challenging owing to the interactions between the light-induced dipoles that can be large when the light is tuned near an atomic resonance. In dilute media, their role can be analyzed using the perturbative approach of Friedberg, Hartmann, and Manassah (FHM) [3], which predicts in particular a "cooperative Lamb shift" measured recently in inhomogeneously broadened media [4,5] and cold dilute atomic gases [6]. For an atom slab, the FHM approach was shown to correspond to the low-density limit of the local-field model introduced by Lorentz [7], which replaces the action of all the atoms of the medium on a particular one by an average effective field [1,2], thus ignoring correlations between the light-induced dipoles. This mean-field approach leads to the Lorentz-Lorenz formula, which allows calculating the index of refraction of many dense media with an excellent accuracy $[1,8]$. However, it was pointed out $[7,9]$ that in the absence of inhomogeneous broadening, such as in cold atomic ensembles, the mean-field response may not be valid due to recurrent scattering where the field radiated by one atom can be scattered back by another atom $[10,11]$. Recurrent scattering should become important when the incident light (wavelength $\lambda=2 \pi / k$ ) is tuned near an atomic resonance, and the atomic density approaches $k^{3}$. This calls for an experiment operating in this regime, where a comparison between the standard mean-field theories of light scattering and a microscopic approach, including recurrent scattering, can be performed.
Here, we perform this comparison. To do so, we need to access a quantity relevant to both the macroscopic and the microscopic approaches. The coherent electric field $\left\langle\mathbf{E}_{\mathrm{sc}}\right\rangle$ scattered by the cloud fulfills this condition: it is obtained by averaging the scattered field $\mathbf{E}_{\mathrm{sc}}$ over many realizations of the spatial random distribution of atoms, and its evolution is governed by the macroscopic Maxwell's equations in the cloud considered as an homogeneous medium described by a susceptibility. In the case of cold atomic gases, the near-resonance coherent optical response has been explored experimentally using mostly dilute, optically thick ensembles [12-19]. Recently, we studied the light scattered by a microscopic dense cloud of cold atoms at $90^{\circ}$ of a near-resonant excitation laser [20]. In that situation, we were sensitive to the incoherent component $\left\langle\left|\mathbf{E}_{\mathrm{sc}}-\left\langle\mathbf{E}_{\mathrm{sc}}\right\rangle\right|^{2}\right\rangle$ of the scattered light. We could therefore not compare our results with mean-field predictions for continuous media, which are only relevant for the coherent part.

In this work, we study the coherent scattering by our microscopic cloud. The cloud contains up to a few hundreds laser-cooled rubidium-87 atoms and has a size smaller than the wavelength of the optical $D_{2}$ transition. We illuminate the sample with a tightly focused laser with a waist larger than the cloud size. We access the coherent scattering by measuring the extinction resulting from the interference of the laser field with the field scattered by the cloud. We observe a saturation of the extinction, a broadening of the line, and a small redshift when we vary the number of atoms from 10 to 180 . We show that the measured shift and width do not agree with the FHM perturbative approach. The description of the atomic cloud by a mean-field model based on the Lorentz-Lorenz 
formula also disagrees with our data. Finally, a microscopic discrete dipole model that incorporates recurrent scattering leads to a qualitatively closer agreement with our measurements, despite remaining differences.

To study the coherent scattering by our cloud, we detect the interference in the far field between the laser field $\mathbf{E}_{\mathrm{L}}$ and the scattered field $\mathbf{E}_{\mathrm{sc}}$. To do so, we use two identical aspherical lenses $L 1$ and $L 2$ with a high numerical aperture $(\mathrm{NA}=0.5)$ mounted in a confocal configuration in a vacuum chamber [see Fig. 1(a)] [21]. $L 1$ focuses far-offdetuned laser light onto a waist of $1.2 \pm 0.1 \mu \mathrm{m}\left(1 / e^{2}\right.$ radius). This creates a dipole trap (depth: $1 \mathrm{mK}$ ) in which we load $N$ atoms with a temperature of $120 \pm 15 \mu \mathrm{K}$ [22]. We control the number of atoms $N$ within $10 \%$ and vary $N$ between 10 and 180 [23]. The atomic cloud is cigar-shaped, with calculated transverse and longitudinal root-meansquare $(\mathrm{rms})$ widths $\left(a_{\perp}, a_{z}\right)=(0.2,1.2) \mu \mathrm{m}$. The peak densities range from $n=10^{13}$ to $2 \times 10^{14} \mathrm{at} / \mathrm{cm}^{3}$. The uncertainties in the temperature, atom number, and waist size lead to a systematic uncertainty on the peak density of a factor of 2 . The probe beam is focused down to a waist of $w=1.20 \pm 0.05 \mu \mathrm{m}$ also by $L 2$ at the position of the cloud. It is linearly polarized and nearly resonant with the closed $D_{2}$ transition of rubidium between the $\left(5 S_{1 / 2}, F=2\right)$ and $\left(5 P_{3 / 2}, F=3\right)$ levels at $\lambda=2 \pi c / \omega_{0}=$ $780.2 \mathrm{~nm}$ (linewidth $\Gamma=2 \pi \times 6 \mathrm{MHz}$ ) [25]. We operate the probe in the low intensity limit where the atoms respond linearly to the field: $I / I_{\text {sat }} \approx 0.04\left(I_{\text {sat }}=1.6 \mathrm{~mW} / \mathrm{cm}^{2}\right)$.

(a)
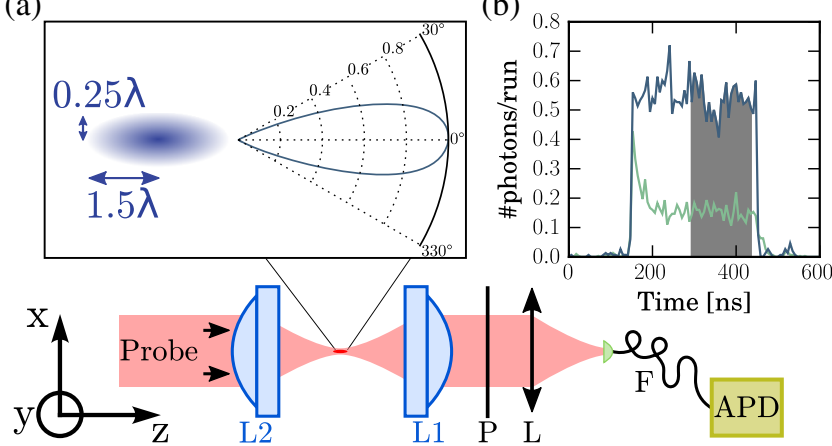

FIG. 1. (a) Experimental setup. A microscopic cloud of ${ }^{87} \mathrm{Rb}$ atoms is illuminated by a linearly polarized probe laser focused down to a waist $w=1.2 \mu \mathrm{m}$. $P$ : polarizer. $L$ : Lens allowing the mode matching between the laser probe beam and the singlemode fiber $(F)$ in the absence of atoms. APD: avalanche photodiode. Inset: cloud rms widths (left) and intensity radiation pattern (right), calculated using a microscopic approach (see text). The coherent part, $\left|\mathcal{E}_{\text {coh }}(\omega)\right|^{2}$, dominates the incoherent part, which is more isotropic and is orders of magnitude smaller. (b) Example of temporal signals recorded on the APD with $N=$ 180 atoms (green line) and without atoms (blue line). The laser is nearly resonant with the atoms, with a frequency detuning $\Delta=0.3 \Gamma$. Each run consists of 1000 illuminations with a duration of $300 \mathrm{~ns}$ each. Temporal bins: 6 ns. Gray area: time interval used for the steady-state analysis.
We collect the probe light transmitted through the cloud using $L 1$ and couple it into a single-mode fiber connected to an avalanche photodiode (APD). The temporal signals are acquired by accumulating single photons using a counting card with a resolution of 150 ps. A polarization beam splitter is placed before the single-mode fiber and aligned at $45^{\circ}$ of the probe laser polarization so as to split the collected light between the fibered APD and a CCD camera [not shown in Fig. 1(a)].

Our configuration is sensitive to the mode matching $\mathcal{E}(\omega)=\int\left\{\mathbf{E}(\mathbf{r}, \omega) \cdot \mathbf{g}^{*}(\mathbf{r})\right\} d S$ between the total field $\mathbf{E}=$ $\mathbf{E}_{\mathrm{L}}+\mathbf{E}_{\mathrm{sc}}$ and the mode $\mathbf{g}$ of the single-mode-fibered detector ( $d S$ is a differential area element perpendicular to the optical axis) [26]. In the absence of atoms, the fiber mode is matched to the incoming light, i.e., $\mathbf{g} \propto \mathbf{E}_{\mathrm{L}}$. In our experiment, we measure $\left\langle|\mathcal{E}(\omega)|^{2}\right\rangle$, where $\langle\cdot\rangle$ means an average over many realizations of the cloud. After averaging, the signal is the sum of two parts [27]: (i) $\left|\mathcal{E}_{\text {coh }}(\omega)\right|^{2}$ due to $\mathbf{E}_{\mathrm{L}}+\left\langle\mathbf{E}_{\mathrm{sc}}\right\rangle$, and (ii) $\left\langle\left|\mathcal{E}_{\text {incoh }}(\omega)\right|^{2}\right\rangle$ due to the fluctuating field $\mathbf{E}_{\mathrm{sc}}-\left\langle\mathbf{E}_{\mathrm{sc}}\right\rangle$. In the direction of propagation of the laser $\left|\mathcal{E}_{\text {coh }}(\omega)\right|^{2} \gg\left\langle\left|\mathcal{E}_{\text {incoh }}(\omega)\right|^{2}\right\rangle$ [see below and in Fig. 1(a)], and we are therefore mainly sensitive to the coherent optical response, which we characterize by a transfer function $\mathcal{S}(\omega)=\langle\mathcal{E}(\omega)\rangle / \mathcal{E}_{\mathrm{L}}(\omega)$ obtained by comparing the detected fields with and without atoms.

To measure $\mathcal{S}(\omega)$ in steady state, we proceed in the following way: after preparing the atoms in the $\left(5 S_{1 / 2}\right.$, $F=2$ ) level, we switch off the dipole trap light during $500 \mathrm{~ns}$ and send a $300 \mathrm{~ns}$ probe pulse with a temporal top hat profile (rise time of $2 \mathrm{~ns}$ ). We then recapture the cloud in the trap for $500 \mathrm{~ns}$ and repeat this release-probe-recapture 1000 times using the same atomic cloud [30]. This procedure is typically repeated with 200 different atomic clouds. A typical signal is shown in Fig. 1(b). It reaches a steady-state after a transient time of $\sim 26 \mathrm{~ns}$, close to the lifetime $1 / \Gamma$ of the excited state, during which the atomic medium gets polarized. We average the signal over a time interval of $120 \mathrm{~ns}$ (gray area) and normalize it with respect to the case without atoms to obtain the transmission in steady state for a given probe frequency. We checked that the scattered light has the same polarization as the probe light by rotating the polarizer $P$ and observing a signal with a contrast of $95 \%$, the same as in the absence of atoms. This feature is characteristic of the coherent scattered field, and therefore confirms experimentally that $\left|\mathcal{E}_{\text {coh }}(\omega)\right|^{2} \gg$ $\left\langle\left|\mathcal{E}_{\text {incoh }}(\omega)\right|^{2}\right\rangle$. Finally, the sequence is repeated for various probe detunings $\Delta=\omega-\omega_{0}$ and atom numbers $N$. We obtain the spectra shown in Fig. 2(a).

The derivation of a functional form for $\mathcal{S}(\omega)$ is very hard in our dense cloud regime. However, in the case of a cloud with a size smaller than $1 / k$ so that it behaves as a small dielectric sphere with a polarizability $\alpha_{\mathrm{c}}(\omega)$ we get, following [26], $\mathcal{S}(\omega) \approx 1+i k \alpha_{\mathrm{c}}(\omega) /\left(\pi w^{2}\right)$, which we cast in the form 

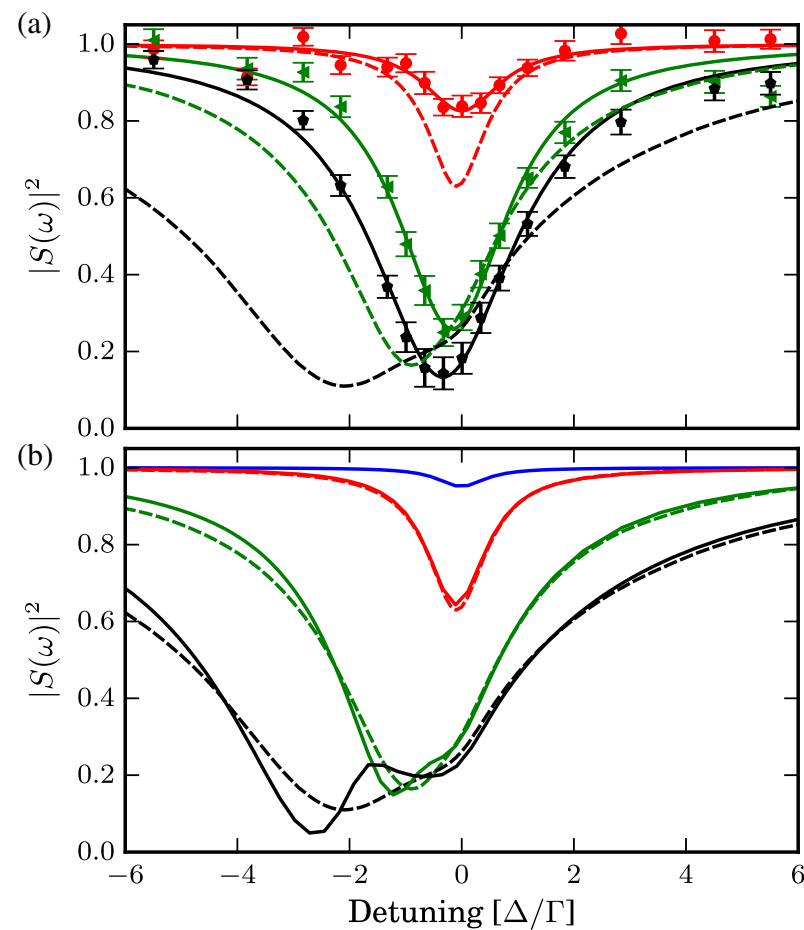

FIG. 2. (a) Measured transfer function of the cloud in steady state versus probe detuning $\Delta$ for $N=(10,83,180)$ atoms (top to bottom); error bars: statistical (one standard deviation), shot noise limited. Solid lines: Lorentzian fit by $|\mathcal{S}(\omega)|^{2}$. Dotted lines: results of the coupled dipole equations including the 12 levels of the $F_{\mathrm{g}}=2-F_{\mathrm{e}}=3$ transition (see text). (b) Comparison between the predictions of the Lorentz model (solid line) and the microscopic, 12-level atom model (dotted line) for $N=$ $(1,10,83,180)$ (top to bottom).

$$
\mathcal{S}(\omega)=1-\frac{A}{1-2 i \frac{\omega-\omega_{c}}{\Gamma_{c}}},
$$

assuming that the polarizability is resonant around a frequency $\omega_{\mathrm{c}}$ with a width $\Gamma_{\mathrm{c}}$. We fit the spectra shown in Fig. 2(a) with the Lorentzian function $|\mathcal{S}(\omega)|^{2}$, using Eq. (1) and leaving $A, \Delta_{c}=\omega_{c}-\omega_{0}$ and $\Gamma_{c}$ as free parameters. The fit agrees well with the data, confirming that the functional form of Eq. (1) is appropriate even for our elongated sample. Figure 3 shows the results of the fits. For increasing atom numbers, we observe a saturation of the amplitude $A$, and therefore of the extinction, an increasing small redshift and a broadening of the line. These behaviors can be understood qualitatively as a consequence of the dipole-dipole interactions between atoms, on the order of $\hbar \Gamma n / k^{3}$ (see below).

We now compare our results to various models of the optical response of the cloud. In Refs. [3,31], Friedberg, Hartmann, and Manassah used perturbation theory to derive the expressions for a collective decay rate and a collective shift for various geometries of an atomic ensemble of two-level atoms. There, the collective shift and

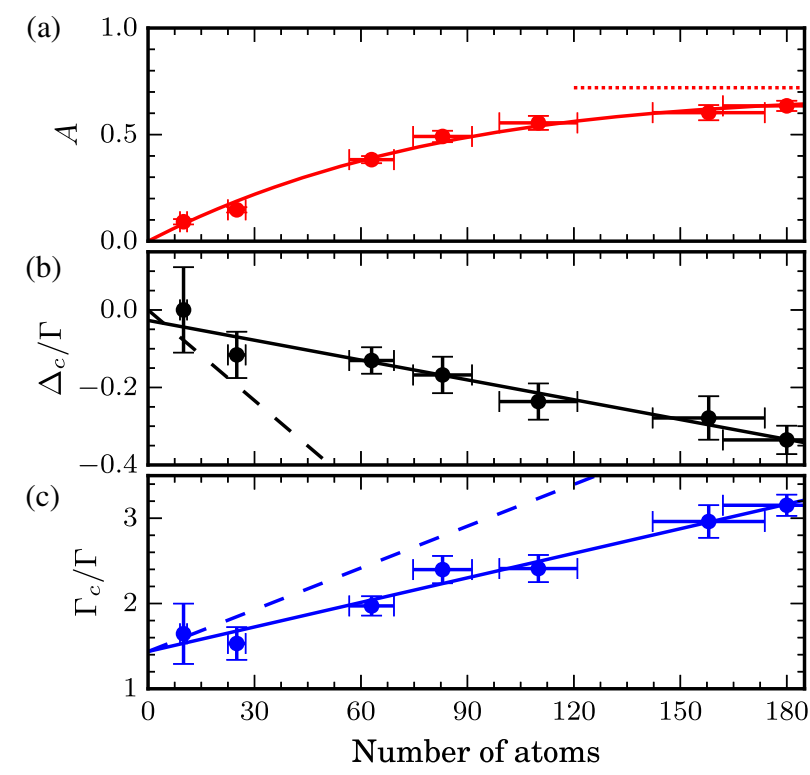

FIG. 3. Fit results of the data of Fig. 2 with the function $|\mathcal{S}(\omega)|^{2}$. Error bars are from the fit. (a) Amplitude $A$. Solid green line: phenomenological fit to guide the eye, yielding a saturation (dotted line) at 0.7. (b) Shift of the center frequency $\Delta_{c}=\omega_{c}-\omega_{0}$. Solid line: linear fit. (c) Full width at half maximum $\Gamma_{c}$. Solid line: linear fit. Dashed lines in (b) and (c): predictions by Friedberg, Hartmann, and Manassah detailed in the text. The prediction for the width has been offset to match the data for $N=0$.

rate are the real and imaginary parts of the average dipole-dipole interaction [32]. This theory predicts the "cooperative Lamb shift" measured in a hot atomic vapor [5] and in a dilute, optically thick cold atomic sample [6]. For the case of an ellipsoidal cloud with Gaussian density distribution, the predictions [see formulas (5.2) and (5.3) of Ref. [31]] are plotted in Figs. 3(b) and 3(c) for our experimental parameters. Here, we included the rubidium internal structure by multiplying the prediction of Ref. [31] by the ratio of multiplicities $7 / 15$ of the $F_{\mathrm{g}}=2-F_{\mathrm{e}}=3$ transition [28,33], assuming equal populations in all hyperfine Zeeman ground states and a negligible magnetic field (as is the case in the experiment) [27]. The predictions differ significantly from the measured values, indicating that this perturbative approach does not apply for our dense, cold atomic systems.

To go beyond the FHM perturbative treatment, we now calculate the optical response as predicted by the Lorentz local field theory for our dense cigar-shaped cloud. For this purpose, we replace the cloud by a Gaussian continuous density distribution $n(\mathbf{r})$ (with rms widths $a_{\perp}$ and $a_{z}$ ) and calculate the local susceptibility using the Lorentz-Lorenz formula $\chi(\mathbf{r}, \omega)=n(\mathbf{r}) \alpha(\omega) /[1-n(\mathbf{r}) \alpha(\omega) / 3]$ [1,2]. Here, $\alpha(\omega)=i(7 / 15)\left(6 \pi / k^{3}\right) /[1-i(2 \Delta / \Gamma)]$ is the polarizability of a single atom, which includes the internal atomic structure of rubidium as described above, see [27]. We then define a local permittivity $\epsilon(\mathbf{r})=1+\chi(\mathbf{r})$ and use a finite 
element program to calculate the electric field scattered in the far field by the cloud illuminated by the Gaussian laser beam. We finally compute the transfer function $\mathcal{S}(\omega)$ taking for the Gaussian field the usual paraxial expression [27]. The results are shown in Fig. 2(b). The mean-field response of the cloud predicted by the Lorentz-Lorenz formula deviates from the data as the number of atoms increases, featuring in particular a double structure for the largest atom numbers [34], as well as a large asymmetry (also observed in the spectrum of transmitted light of an atomic slab described by the Lorentz-Lorenz formula [7]).

We finally calculate the coherent response of the cloud using a microscopic model where the atoms are considered as pointlike dipoles $\mathbf{d}_{j}$ randomly positioned according to the Gaussian spatial distribution, each being driven by the laser field and the fields scattered by all the other ones $[11,35]$. This approach leads to a set of coupled dipole equations. As in Ref. [20], we include the internal structure of the atoms by randomly assigning them a given Zeeman state $m_{j}$ of the $\left(5 S_{1 / 2}, F=2\right)$ manifold, and we write $\mathbf{d}_{j}=\mathcal{D} \sum_{\sigma} \hat{\mathbf{e}}_{\sigma} C_{m_{j}}^{(\sigma)} \mathcal{P}_{j \sigma}(\sigma= \pm 1,0$ defines the polarization $)$ The amplitude of the atomic dipole $j$ associated to the optical transition $\left|g, m_{j}\right\rangle \rightarrow\left|e, m_{j}+\sigma\right\rangle$ is proportional to the reduced dipole matrix element $\mathcal{D}$, the atomic coherence $\mathcal{P}_{j \sigma}$, and the corresponding Clebsch-Gordan coefficient $C_{m_{j}}^{(\sigma)}$. We solve the steady state set of coupled equations for the coherences

$$
(\Delta+i \Gamma / 2) \mathcal{P}_{j \alpha}=\Omega_{j \alpha}+\sum_{l \neq j} \sum_{\beta} C_{m_{l}}^{(\beta)} C_{m_{j}}^{(\alpha)} V_{j \alpha}^{l \beta}(\mathbf{r}) \mathcal{P}_{l \beta},
$$

where $\quad V_{j \alpha}^{l \beta}=-V_{\mathrm{dd}}\left[p_{\alpha \beta}(i k r-1)+q_{\alpha \beta}(k r)^{2}\right] e^{i k r} \quad$ with $V_{\mathrm{dd}}=3 \hbar \Gamma / 4(k r)^{3}$ is the dipole-dipole interaction, $p_{\alpha \beta}$ and $q_{\alpha \beta}$ are angular functions [20], and $\Omega_{j \alpha}$ the Rabi frequency. We calculate the field scattered by the cloud [yielding the radiation pattern shown in Fig. 1(a)]. We then compute the interference of this field with the laser field, at the position of the lens $L 1$, and the transfer function for this particular configuration of the atomic ensemble and average over many spatial configurations.

The results of the microscopic model are plotted in Fig. 2(b) for various detunings and atom numbers, together with the prediction of the Lorentz local field model. We observe that both models are in agreement for low values of $N$, and predict approximately Lorentzian line shapes. For large atom numbers, however, they differ quantitatively, pointing towards the role of recurrent scattering, included in the microscopic model, to all orders, but not in the Lorentz model [9-11,36,37]. To the lowest order in density, for a cloud (density $n$ ) of identical atoms with polarizability $\alpha$, the contribution of recurrent scattering to the susceptibility is proportional to the number of atom pairs $(n \alpha)^{2}$ inside the scattering volume $\alpha$. It becomes important when $n \alpha \sim 1$. The onset of light-induced correlations and the effect of recurrent scattering as a function of the detuning and atom density was analyzed in more detail in $[7,10,11,38,39]$. In the presence of recurrent scattering and when $n / k^{3} \ll 1$, the susceptibility takes the form

$$
\chi(\omega)=\frac{n \alpha(\omega)}{1-n \alpha(\omega)\left[\frac{1}{3}+\beta(\omega)\right]},
$$

where $\beta(\omega)$ is the contribution from recurrent scattering. Using formula (22) of [10], we get $\beta(\omega) \propto B \alpha(\omega) k^{3}$, with $B$ a volume integral challenging to calculate for our geometry. The lowest order contribution to $\beta$ is independent of the density. If $B \sim 1$, the local field correction $n \alpha / 3$ is thus on the same order as the recurrent scattering contribution close to resonance $\left(\alpha=6 \pi i / k^{3}\right)$, while away from resonance $\left(\alpha k^{3} \ll 1\right)$, the influence of recurrent scattering is negligible. Remarkably, when inhomogeneous broadening is introduced (such as the Doppler effect in hot vapor cells [5]), the resonant frequencies of the dipoles $j$ are spread over $\Delta \omega_{\mathrm{D}}$, and $\beta$ is replaced by the average over $j$, $\langle\alpha\rangle_{j} k^{3}$, and is therefore reduced by a factor $\Gamma / \Delta \omega_{\mathrm{D}}$ [9]. This explains why for any medium where inhomogeneous broadening is dominant the Lorentz-Lorenz model is successful, as $\Gamma / \Delta \omega_{\mathrm{D}} \ll 1$ and thus, $\beta \ll 1$. On the contrary, in the absence of inhomogeneous broadening, the Lorentz-Lorenz formula is usually not valid at resonance. In [40], we use the microscopic approach to calculate the effective dielectric constant of our cloud, but in the regime $n / k^{3} \geq 1$, and found that it does not follow the Lorentz-Lorenz formula, as expected.

Finally, we compare our measurements to the microscopic model [see Fig. 2(a)]. We observe that the data are closer to this model than to the Lorentz model, as they do not show the double structure predicted by the Lorentz model for the largest atom numbers. This indicates that the Lorentz model is not valid in our configuration. However, the measurements exhibit systematically less pronounced features for the shift, width, and amplitude than predicted by the microscopic model. On the experimental side, we have ruled out possible biases, such as the probe beam alignment [27] and the possible cumulative heating of the cloud due to the pulsed illumination that could result in a modification of the cloud volume. On the theoretical side, the models ignore quantum fluctuations between hyperfine ground states and assume the low light intensity limit, which may in practice be difficult to fully realize in the experiments due to secondary radiation by closely spaced atoms.

As a conclusion, we have measured the coherent scattering by a dense, cold atomic cloud. We have observed a failure of standard models, such as the FHM model or the mean-field Lorentz model. The remaining difference with the microscopic model shows that a quantitative understanding of the light-induced interactions even in a relatively simple situation is still a challenge. 
We thank C. S. Adams for discussions. We acknowledge support from the EU (ERC Starting Grant ARENA and the HAIRS project), from the Region Ile-de-France (LISCOLEM project), the EPSRC, and the Leverhulme Trust. N. J. S is supported by Triangle de la Physique and S. J. by labex PALM (ECONOMIC project). J.-J. G. acknowledges the support of Institut Universitaire de France.

*Corresponding author. antoine.browaeys@institutoptique.fr

[1] M. Born and E. Wolf, Principle of Optics, 7th ed. (Cambridge University Press, Cambridge, UK, 1999).

[2] D. J. Jackson, Classical Electrodynamics, (John Wiley and Sons, New York, 1998).

[3] R. Friedberg, S. R. Hartmann, and J. T. Manassah, Frequency shift and absorption by resonant systems of two-level atoms, Phys. Rep. 7, 101 (1973).

[4] R. Röhlsberger, K. Schlage, B. Sahoo, S. Couet, and R. Roeffer, Collective Lamb shift in single-photon superradiance, Science 328, 1248 (2010).

[5] J. Keaveney, A. Sargsyan, U. Krohn, I. G. Hughes, D. Sarkisyan, and C. S. Adams, Cooperative Lamb shift in an atomic vapor layer of nanometer thickness, Phys. Rev. Lett. 108, 173601 (2012).

[6] S. J. Roof, K. J. Kemp, M. D. Havey, and I. M. Sokolov, Observation of single-photon superradiance and the cooperative Lamb shift in an extended sample of cold atoms, arXiv:1603.07268.

[7] J. Javanainen and J. Ruostekoski, Light propagation beyond the mean-field theory of standard optics, Opt. Express 24, 993 (2016).

[8] S. E. Schnatterly and C. Tarrio, Local fields in solids: microscopic aspects for dielectrics, Rev. Mod. Phys. 64, 619 (1992).

[9] J. Javanainen, J. Ruostekoski, Y. Li, and S.-M. Yoo, Shifts of a resonance line in a dense atomic sample, Phys. Rev. Lett. 112, 113603 (2014).

[10] O. Morice, Y. Castin, and J. Dalibard, Refractive index of a dilute Bose gas, Phys. Rev. A 51, 3896 (1995).

[11] J. Ruostekoski and J. Javanainen, Quantum field theory of cooperative atom response: Low light intensity, Phys. Rev. A 55, 513 (1997).

[12] H. Bender, C. Stehle, S. Slama, R. Kaiser, N. Piovella, C. Zimmermann, and Ph. W. Courteille, Observation of cooperative Mie scattering from an ultracold atomic cloud, Phys. Rev. A 82, 011404 (2010).

[13] T. Bienaimé, S. Bux, E. Lucioni, Ph. W. Courteille, N. Piovella, and R. Kaiser, Observation of a cooperative radiation force in the presence of disorder, Phys. Rev. Lett. 104, 183602 (2010)

[14] J. Chabé, M.-T. Rouabah, L. Bellando, T. Bienaimé, N. Piovella, R. Bachelard, and R. Kaiser, Coherent and incoherent multiple scattering, Phys. Rev. A 89, 043833 (2014).

[15] M. Chalony, R. Pierrat, D. Delande, and D. Wilkowski, Coherent ash of light emitted by a cold atomic cloud, Phys. Rev. A 84, 011401 (2011).
[16] C. C. Kwong, T. Yang, M. S. Pramod, K. Pandey, D. Delande, R. Pierrat, and D. Wilkowski, Cooperative emission of a coherent superflash of light, Phys. Rev. Lett. 113, 223601 (2014).

[17] S. Roof, K. Kemp, M. Havey, I. M. Sokolov, and D. V. Kupriyanov, Microscopic lensing by a dense, cold atomic sample, Opt. Lett. 40, 1137 (2015).

[18] S. L. Bromley et al., Collective atomic scattering and motional effects in a dense coherent medium, Nat. Commun. 7, 11039 (2016).

[19] M. O. Araújo, I. Kresic, R. Kaiser, and W. Guérin, Superradiance in a large cloud of cold atoms in the linear-optics regime, arXiv:1603.07204.

[20] J. Pellegrino, R. Bourgain, S. Jennewein, Y. R. P. Sortais, A. Browaeys, S. D. Jenkins, and J. Ruostekoski, Observation of suppression of light scattering induced by dipole-dipole interactions in a cold atom ensemble, Phys. Rev. Lett. 113, 133602 (2014).

[21] Y. R. P. Sortais et al., Diffraction-limited optics for singleatom manipulation, Phys. Rev. A 75, 013406 (2007).

[22] The Doppler broadening $(k \Delta v=0.06 \Gamma)$ is negligible with respect to the linewidth $\Gamma$.

[23] The exact number of atoms inside the trap varies shot to shot as it is governed by a sub-Poisson distribution with a mean $N$ and variance $3 N / 4$ [24].

[24] Y. R. P. Sortais, A. Fuhrmanek, R. Bourgain, and A. Browaeys, Sub-Poissonian atom-number fluctuations using light-assisted collisions, Phys. Rev. A 85, 035403 (2012).

[25] The magnetic field is compensated to values lower than $\sim 80 \mathrm{mG}$

[26] S. A. Aljunid, M. K. Tey, B. Chng, T. Liew, G. Maslennikov, V. Scarani, and C. Kurtsiefer, Phase shift of a weak coherent beam induced by a single atom, Phys. Rev. Lett. 103, 153601 (2009).

[27] See Supplemental Material at http://link.aps.org/ supplemental/10.1103/PhysRevLett.116.233601, which includes Refs. [2,11,17,28,29].

[28] J. Ruostekoski, Scattering of light and atoms in a FermiDirac gas with Bardeen-Cooper-Schrieer pairing, Phys. Rev. A 61, 033605 (2000).

[29] J. Ruostekoski and J. Javanainen, Optical linewidth of a low density Fermi-Dirac gas, Phys. Rev. A 56, 2056 (1997).

[30] Our results vary by less than $5 \%$ when the same cloud is illuminated with a single shot probe. This observation rules out a possible cumulative modification of the cloud volume during the pulsed excitation.

[31] J. T. Manassah, Cooperative radiation from atoms in different geometries: decay rate and frequency shift, Adv. Opt. Photonics 4, 108 (2012).

[32] M. O. Scully, Collective Lamb Shift in Single Photon Dicke Superradiance, Phys. Rev. Lett. 102, 143601 (2009).

[33] C. A. Müller, T. Jonckheere, Ch. Miniatura, and D. Delande, Weak localization of light by cold atoms: The impact of quantum internal structure, Phys. Rev. A 64, 053804 (2001).

[34] The double structure appearing in the Lorentz model indicates that the resonance of the coherent response of the cloud is not only related to the resonance of the susceptibility, but also to a shape resonance. 
[35] L. Chomaz, L. Corman, T. Yefsah, R. Desbuquois, and J. Dalibard, Absorption imaging of a quasi-twodimensional gas: a multiple scattering analysis, New J. Phys. 14, 055001 (2012).

[36] A. Lagendijk and B. A. van Tiggelen, Resonant multiple scattering of light, Phys. Rep. 270, 143 (1996).

[37] I. M. Sokolov, M. D. Kupriyanova, D. V. Kupriyanov, and M. D. Havey, Light scattering from a dense and ultracold atomic gas, Phys. Rev. A 79, 053405 (2009).
[38] R. Saunders and R. K. Bullough, Perturbation theory of super-radiance II. Cooperative and non-cooperative level shifts, J. Phys. A 6, 1360 (1973).

[39] J. Ruostekoski and J. Javanainen, Optical Linewidth of a Low Density Fermi-Dirac Gas, Phys. Rev. Lett. 82, 4741 (1999).

[40] N. J. Schilder, C. Sauvan, J.-P. Hugonin, S. Jennewein, Y. R. P. Sortais, A. Browaeys, and J.-J. Greffet, Polaritonic modes in a dense cloud of cold atoms, arXiv:1510.07993v2. 